\title{
CHC ALGORITHM FOR ANTENNA ARRAY WITH FAILED ELEMENTS OPTIMIZATION
}

\author{
Bogdan Artyushenko, Galina Shilo, Volodymyr Krischuk \\ Zaporizhzhya National Technical University \\ Zhukovsky Str. 64, Zaporizhzhya, Ukraine, 69063 \\ e-mail: bogdartysh@ukr.net, gshilo@zntu.edu.ua
}

\begin{abstract}
A new CHC based method for optimization of antenna array, with failed elements, is developed. Performances of $\mathrm{CHC}$ and canonical genetic algorithm are compared. Numerical results show that CHC gives better results with the same working time. To improve computational efficiency parallelization possibilities are studied.
\end{abstract}

Keywords: Genetic algorithm, parallel computation, tolerable design, tolerance allocation, antenna array.

\section{INTRODUCTION}

Antenna array is subjected to failures of its modules and deviations. Element failures destroy symmetry, cause sharp variations in the field intensity, increase side lobe levels. Failure of one element with high amplitude leads, in general, to unacceptable changes of antenna characteristics. Amplitudes and phases of remaining antenna array elements are to be redistributed to overcome these changes of antenna array departure characteristics. In this way real-time restoration could be achieved.

Numerous methods have been proposed to reduce pattern diagram shift when several elements fail [1], [2]. These approaches assume, that remaining elements have rating values. But working elements' parameters often deviate when some elements fail. So these methods have limits for practical use. To overcome these limitations genetic algorithm (GA) based methods could be used. The main advantages of methods based on GA are: they could be used as a uniform approach for different antenna types with elements failures [3], when parameters deviate [4]; continuity of parameter space is not required; parallelization is easily implemented; reduction of the chance of being trapped into a local optimum.

Estimation of antenna array far-field diagram (pattern) characteristic is computationally hard. Therefore a GA with small number of recalculations is needed. One of spread GA, that satisfies this requirement, is $\mathrm{CHC}$ [5].

The aim of this paper is to develop CHC-based approach of optimization for antenna array with failed elements.
To reach the aim it is necessary

- $\quad$ to consider the criteria of antenna array far field diagram optimality;

- $\quad$ to modify genetic algorithms for antenna array optimization;

- to compare results of canonical GA and $\mathrm{CHC}$;

- $\quad$ to consider parallelization possibilities.

\section{OPTIMALITY CRITERIA OF ANTENNA ARRAY WITH FAILED ELEMENTS}

It this paper uniformly spaced planar antenna arrays of unidirectorial elements (Fig. 1) is studied. If an element transmits no power, it is considered as failed. No mutual coupling effects are modeled; so failed elements are just deleted from the array factor. Let $N_{x}, N_{y}$ denote the number of columns and rows, than the far-field diagram is given by

$$
E_{\theta \varphi}=\sum_{n x=1}^{N_{x}} \sum_{n y=1}^{N_{y}} I_{n_{x}, n_{y}} e^{j\left(n_{x} k_{x} d_{x}+n_{y} k_{y} d_{y}\right)},
$$

where $k_{x}=\frac{2 \pi}{\lambda} \sin \theta \cos \varphi, k_{y}=\frac{2 \pi}{\lambda} \sin \theta \sin \varphi ;$ $I_{n_{x}, n_{y}}=A_{n_{x}, n_{y}} e^{\sqrt{-1 \cdot v_{n_{x}, n_{y}}}}, A_{n_{x}, n_{y}}, v_{n_{x}, n_{y}}$ are excitation, amplitude and phase of the element $\left(n_{x}, n_{y}\right)$; $d_{x}, d_{y}$ are distances between elements. 


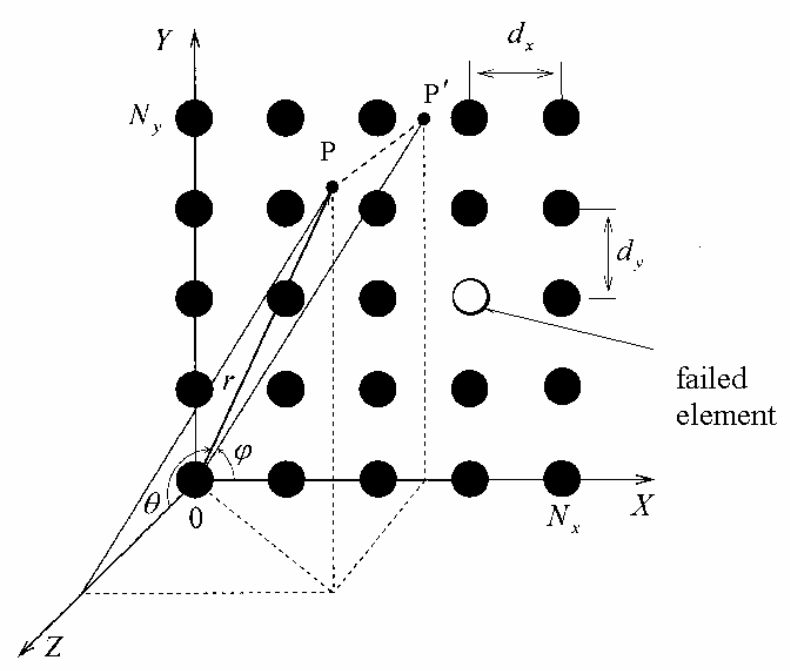

Fig. 1 - Antenna array with failed element

The task is to recover far-field diagram characteristics, i.e. to approach far-field diagram of antenna array with failed element to nominal diagram and to decrease side lobe level. To estimate usefulness of antenna its integral characteristic of far-field diagram is used. So the objective function is to minimize differences between obtained and nominal diagrams at required angles

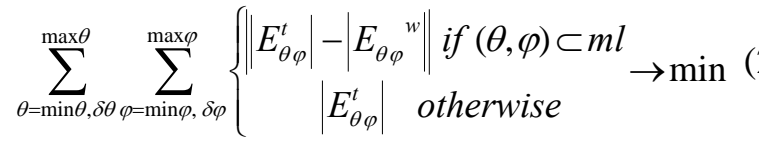

subject to

$$
\begin{gathered}
A_{n_{x}, n_{y}}^{t}=A_{n_{x}, n_{y}}^{w}+d A_{n_{x}, n_{y}}, \\
A_{n_{x}, n_{y}}^{t}=0 \quad \text { if }(n x, n y) \subset F, \\
\frac{d A_{n_{x}, n_{y}} \leq d A_{n_{x}, n_{y}} \leq \overline{d A_{n_{x}, n_{y}}},}{\frac{v_{n_{x}, n_{y}}^{t}}{d}=v^{w}{ }_{n_{x}, n_{y}}+d v_{n_{x}, n_{y}},} \\
\frac{d v_{n_{x}, n_{y}}}{} \leq d v_{n_{x}, n_{y}} \leq \overline{d v_{n_{x}, n_{y}}}
\end{gathered}
$$

$$
S L L<\max S L L
$$

where $A_{n_{x}, n_{y}}^{w}, v_{n_{x}, n_{y}}^{w}, E_{\theta \varphi}{ }^{w}$ are amplitudes, angles and diagram of normal working antenna array; $A_{n_{x}, n_{y}}^{t}, \quad v_{n_{x}, n_{y}}^{t}, E_{\theta \varphi}{ }^{t}$ are amplitudes, phases and diagram of antenna array with failed elements; $d A_{n_{x}, n_{y}}, \quad d v_{n_{x}, n_{y}}$ are redistributed additional amplitudes and phases;

$d A_{n_{x}, n_{y}}, \overline{d A_{n_{x}, n_{y}}}, d v_{n_{x}, n_{y}} \overline{d v_{n_{x}, n_{y}}}$ are minimal and maximal possible additional amplitudes and phases; $(\min \varphi, \max \varphi),(\min \theta, \max \theta)$ are ranges of examined angles in horizontal and vertical fields; $\delta \varphi, \delta \theta$ are steps of angle change (e.g. $1^{0}$ ); $m l=(\underline{m l}, \overline{m l})$ is main lobe; $F$ is a set of failed elements; $\quad S L L$ is side lobe level; $\max S L L$ is maximal permissible value of side lobe level.

If parameters of working elements deviate, $E_{\theta \varphi}^{t}$ is interval extension of far-field diagram[4], and equation 2 should be replaced with

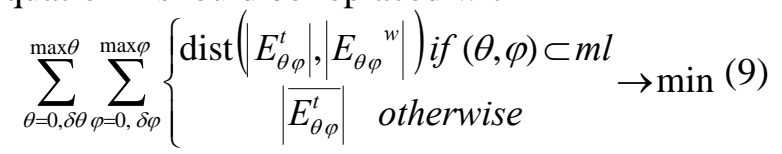

where dist is Hemisdorf distance and $\left|\overline{E_{\theta \varphi}^{t}}\right|$ is supreme value of interval extension of complex function modulus.

Optimal solution is to be found by genetic algorithm. This solution is redistributed additional amplitudes and phases of working elements.

\section{GENETIC ALGORITHM AND CHC}

In canonical GA (CGA) each genome (solution) of population (set of solutions) is represented as a bit string. During each successive stage several steps are executed. At first some solutions of the current generation's population are selected to breed a new generation according to the objective function (equations 2,9). The next step is to generate the following generation population from selected solutions through genetic operators: crossover (also called recombination), and/or mutation. Crossover is applied to randomly paired solutions (parents) to form new solutions (children, offspring). CGA crossover divides parents' bit strings in two and crosses them to create a child. So bits depend on each other and on parents. Mutation adds random changes to children.

To use GA for antenna array design several changes should be made to canonical GA. So as computing of antenna departure diagram is time consuming task, elitism mechanism should be used. We need a GA, which stores the best-found solutions, and prevents from recalculation of identical solutions. The initial population shouldn't be generated in random. To obtain initial solutions the standard methods of antenna adaptation [2] should be used. Mutation procedure should be applied to diverse these solutions.

CHC (Cross generational elitist selection Heterogeneous recombination (incest preventing) and Cataclysmic mutation) is one of GA that can be used with these conditions. Steps of CHC are similar to GA. But after recombination, the best unique parents and children create the next generation. So 
the best bit strings are copied from one population to following ones. The CHC uses cataclysmic mutation (very heavy mutation to increase diversity), uniform crossover (each bit of the child string doesn't depend on the rest child's bits) and random selection, but prevents incest (selection of very close genomes) and duplications. CHC also uses special mechanism to prevent population from failing in one of local minimums.

Figs. 2,3 show basic stages of CGA and CHC (with insignificant simplifications).

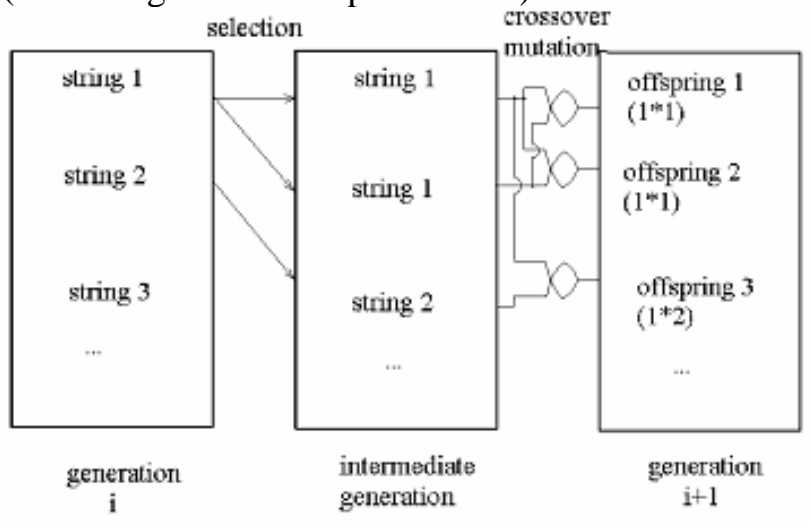

Fig. 2 - Basic stage of CGA

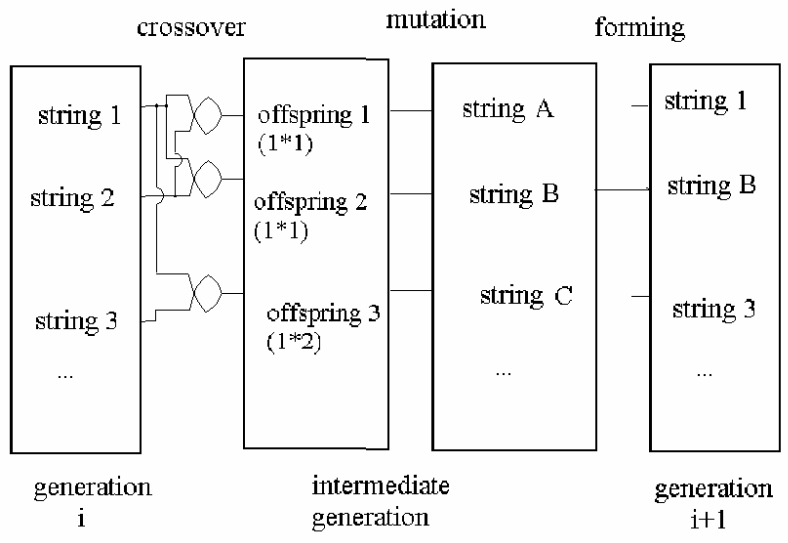

Fig. 3 - Basic stage of CHC

Special encoding of parameters as bit string should be developed for using CHC and CGA as antenna array optimizing approaches. Redistributed additional amplitudes and phases of elements are encoded as one bit string (genome) $G$. Encoding formulae are written by

$$
\begin{aligned}
d v_{n_{x}, n_{y}}=\sum_{l=0}^{L} & 2^{-l-1} G_{L \cdot\left(\left(n_{y}-1\right) N_{x}+n_{x}\right)+l} \times \\
& \times w_{n_{x}, n_{y}}^{v}+\underline{d v_{n_{x}, n_{y}}}, \\
d A_{n_{x}, n_{y}}=\sum_{l=0}^{L} & 2^{-l-1} G_{L \cdot\left(\left(N_{y}+n_{y}-1\right) N_{x}+n_{x}\right)+l} \times \\
& \times w_{n_{x}, n_{y}}^{A}+d A_{n_{x}, n_{y}},
\end{aligned}
$$

where $L$ is a length of substring, which represents one parameter (e.g. $d A_{n_{x}, n_{y}}, d v_{n_{x}, n_{y}}$ );

$$
\begin{aligned}
& w_{n_{x}, n_{y}}^{v}=\left(\overline{d v_{n_{x}, n_{y}}}-d v_{n_{x}, n_{y}}\right) ; \\
& w_{n_{x}, n_{y}}^{A}=\left(\overline{d A_{n_{x}, n_{y}}}-d A_{n_{x}, n_{y}}\right) .
\end{aligned}
$$

\section{PARALLELIZATION}

For CHC parallelization two schemes are mostly used pCHC and Island pCHC [6]. The first one is simpler in realization and more efficient [6].

In pCHC the processors are divided in two groups: several clients (they calculate) and one server (it prepares data and arrange communications). So as calculating of fitness function takes nearly all the time, the clients should calculate primary fitness and server process should do CHC procedure, organize data flow.

For the task of antenna array adaptation a lot of solutions have too high side lobe level. So for these solutions the full integral characteristic of departure diagram isn't needed, and calculation of SLL will be enough. It means that time of calculation decreases for some solutions. And as a result time of objective function calculation deviates. So the optimal number of genomes which one processor calculates on one step should be found. That is: all $N_{c p u}$ client processors are given equal portions of population $\left(2 * N_{\text {perone }}\right.$ parent genomes) to calculate, then those, which have calculated earlier, are given another portion until all genomes of population are calculated. And, with optimal $N_{\text {perone}}$, the wasted time on waiting results of slower processor will be minimal.

Nowadays real-time operating systems such as QNX Neutrino with computer network (control systems and operators' workdesks) are widely used for radar systems. The advantages of QNX network system qnet for parallel computations are: the program is the same for network and one-computer implementation, delays are approximately as long as sockets over TCP/IP, programs can be easily started on another computer, and while MPI allows sending only data of one type, qnet gives a possibility of sending data of different types thus reducing number of data transfer.

In proposed QNX realization (Fig. 4) of CHC client processes are easily started and stopped on all computers (and on server) by remote command, and there is no need for clients to wait until server process stops calculating its part of genomes: there will be two process (threads) for this task. So as computer network is subject to failures, server process should just send $N_{\text {perone }} * 2$ parent genes to whatever asks, and receive the results on the same step (using standard QNX server's calls). 


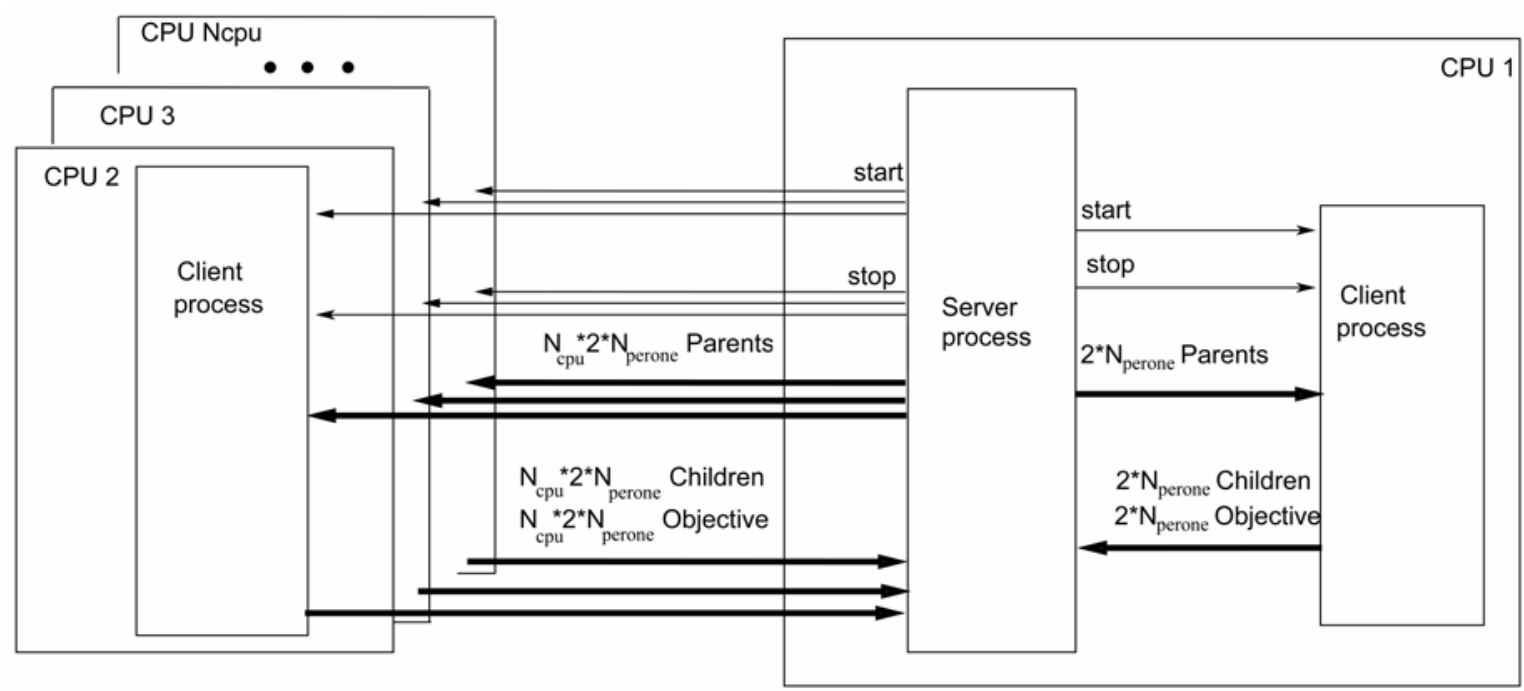

signal

data

Fig. 4 - System architecture of parallel GA on PC network

If server founds that some tasks take too much time (it can mean that client processors are down), it sends it to another clients. If then server receives results from one of such clients it stops calculations on the duplicating processors and gives them some other tasks. Clients are started independently and blocked on sending empty results, when server reads these results it answered with tasks, clients solve them, and are blocked again on sending results of calculations (children genomes and theirs fitness's results) then circle repeats. In this way server doesn't depend on clients, can work with new clients and without failed ones.

\section{EXAMPLES}

The optimization of $21 \times 21$ element planar antenna array [7] with distances $d_{x}=d_{y}=0.6 * \lambda$ and excitation of elements is given by

$$
\begin{gathered}
A_{n_{x}, n_{y}}^{w}=\left[\frac{2}{25}+\frac{23}{25} \cos ^{2}\left(\frac{\pi}{2} \cdot \frac{\left|n_{x}-\frac{N_{x}}{2}\right|}{\frac{N_{x}}{2}}\right)\right] \times \\
\times\left[\frac{2}{25}+\frac{23}{25} \cos ^{2}\left(\frac{\pi}{2} \cdot \frac{\left|n_{y}-\frac{N_{y}}{2}\right|}{\frac{N_{y}}{2}} \mid\right],\right. \\
v_{n_{x}, n_{y}}^{w}=0 .
\end{gathered}
$$

When central element fails $(F=\{(11,11)\})$ the amplitude far-field diagram changes rapidly (Figs. 5,6). This antenna array has $-40 \mathrm{~dB}$ side lobe level. Let us consider planes $\varphi=0, \varphi=\pi / 4$, $\varphi=\pi / 2$ and parameters of equations 2,9 are $\max \varphi=\pi / 2, \quad \delta \varphi=\pi / 4, \quad \max \theta=\pi, \delta \theta=1^{\circ}$. Only elements $6 \leq n_{x} \leq 16,6 \leq n_{y} \leq 16$ can be changed. Constraints are $d v_{n_{x}, n_{y}} \leq 0.5,0 \leq d A_{n_{x}, n_{y}} \leq 0.5$.

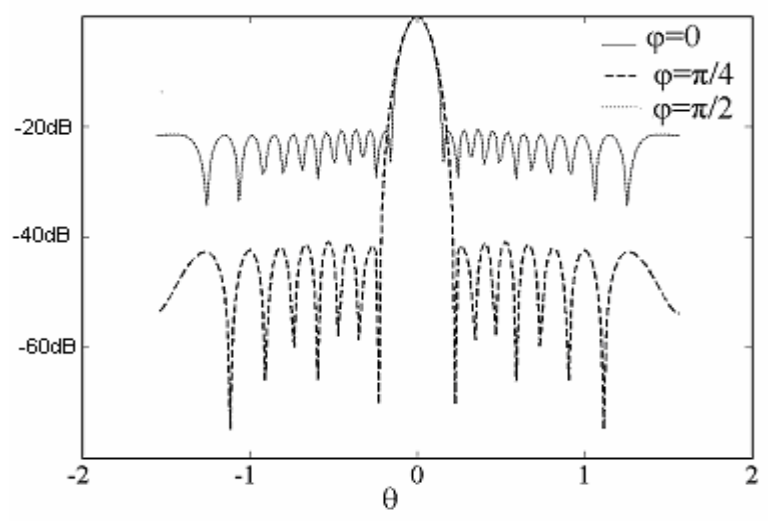

Fig. 5 - Far-field diagram of nominal antenna array

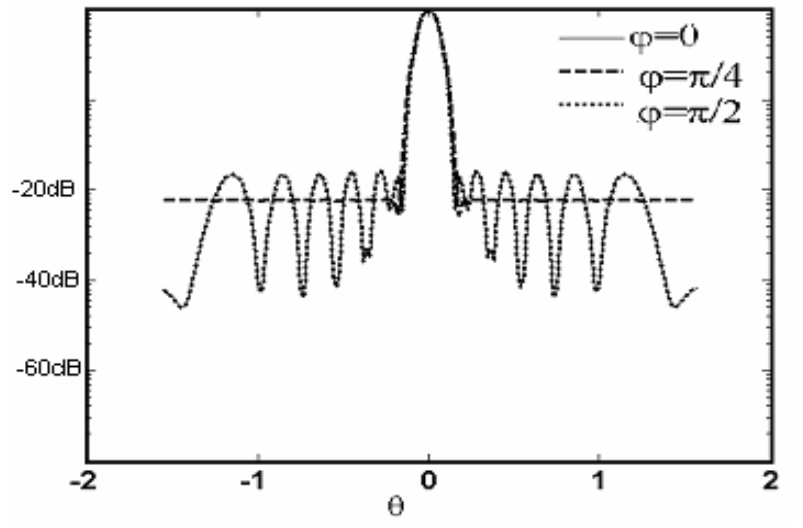

Fig. 6 - Far-field diagram of antenna array with failed central element 
Figs. 7,8 show that canonical genetic algorithm (population size -120 , elite - 2, single point crossover, roulette selection) gives worse results then CHC (population size - 60, uniform crossover and mutation).

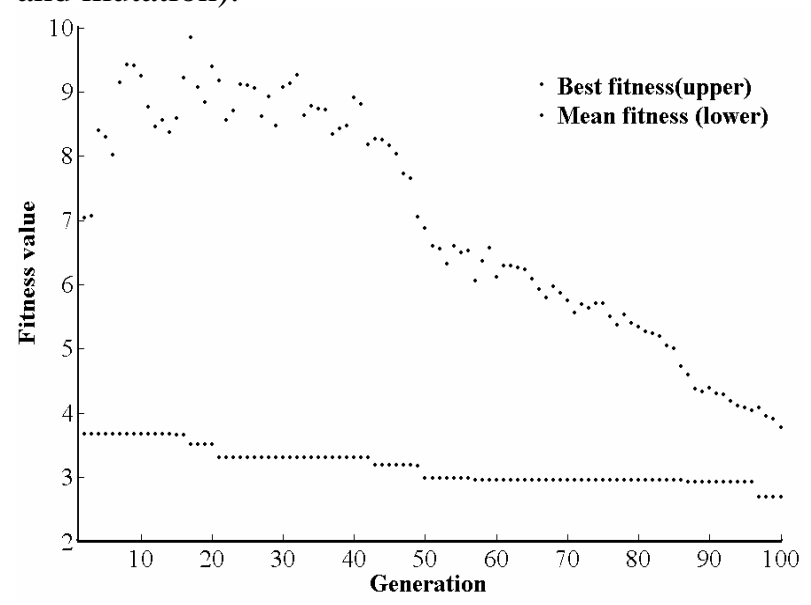

Fig. 7 - Process of canonical genetic algorithm

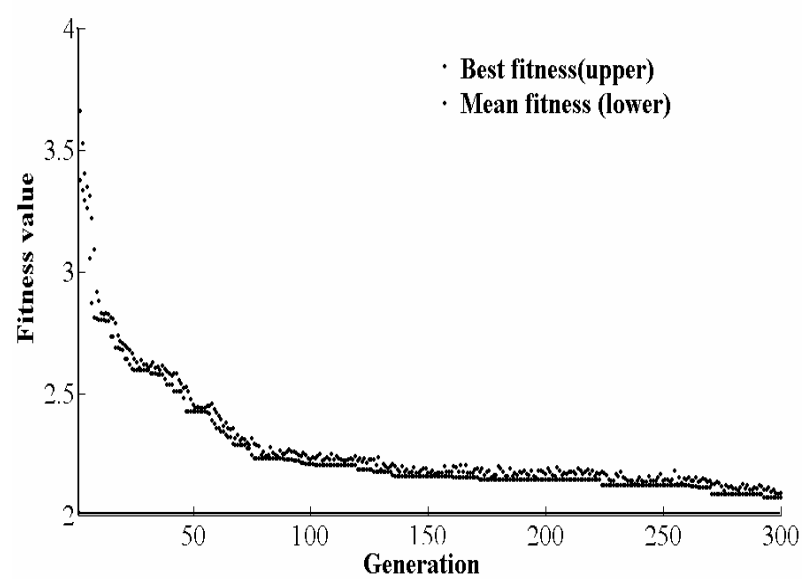

Fig. 8 - Process of CHC

A lot of tests have been done to estimate influences of CGA parameters; they have shown that current parameters of $\mathrm{CHC}$ are close to optimal. For CHC $40 * 300$ and for CGA $120 * 100$ estimations of genomes were made. CHC gives best objective function (equation 2) value approximately 2.04 and canonical GA gives 2.95. These data corresponds to numerical results when 2, 3, 4 random elements are failed.

Figs. 9,10 show the far-field diagram of antenna array optimized with CHC and GA. So as the task is to optimize all three planes $(\varphi=0, \varphi=\pi / 4$, $\varphi=\pi / 2$ ), side lobe level is very high.

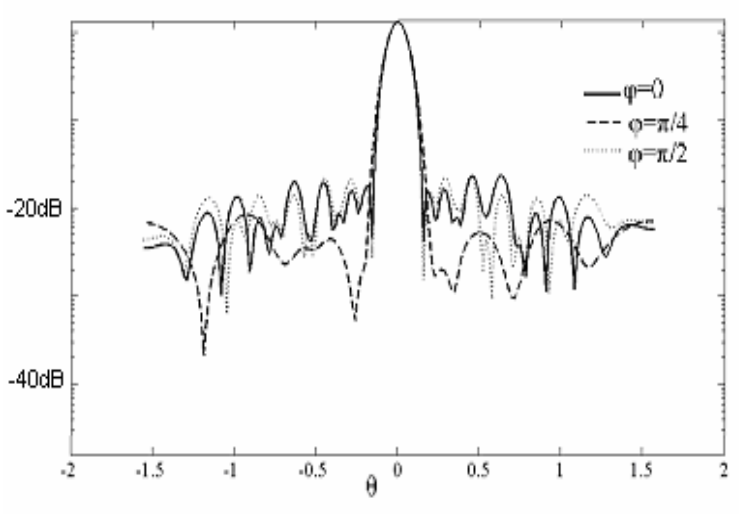

Fig. 9 - Far-field diagram of antenna optimized by canonical genetic algorithm

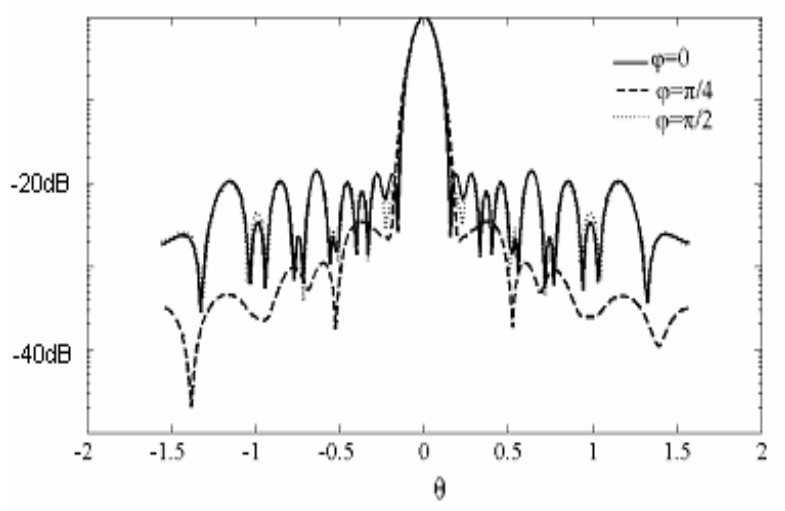

Fig. 10 - Far-field diagram of antenna optimized by CHC

Figs. 11,12 show redistribution of amplitude and phase, which was found by $\mathrm{CHC}$ as optimal for farfield characteristics recovery. As can be seen CHC have produced more complicated redistribution than analytical methods [2,6]. More elements are used, and their power changes not so significantly as in [6], so these results are more stable in term of working conditions of remaining elements.

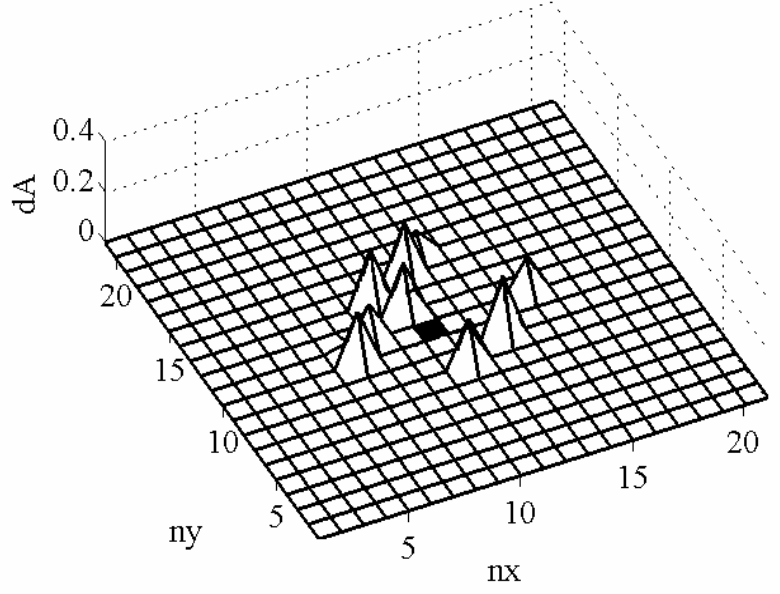

Fig. 11 - Amplitude redistribution 


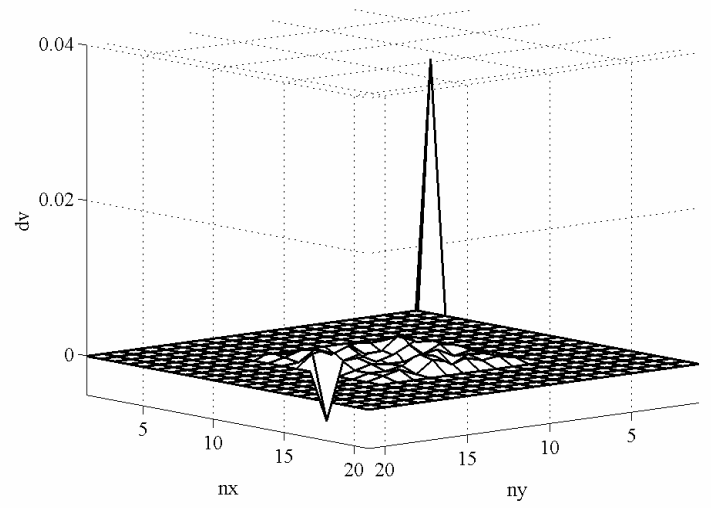

Fig. 12 - Phase redistribution

Table 1 shows time of calculation on $\mathrm{CHC}$ average stage with CPU AMD Athlon $1.66 \mathrm{GHz}$ and 256MB memory. As can be seen, calculation of fitness function needs nearly all the time, and fluctuates a lot (deviation is $20 \%$ ).

Table 1. Time distribution of CHC step

\begin{tabular}{|c|c|}
\hline Step & Time \\
\hline Selection+Reproduction & 0.01 \\
\hline Crossover+Mutation & 0.001 \\
\hline Fitness calculation & $0.235 \pm 20 \%$ \\
\hline
\end{tabular}

Time of one CHC stage calculation by network of 3 computers (AMD Athlon $1.66 \mathrm{GHz}$, memory $256 \mathrm{MB}$, Fast Ethernet) and by cluster of Glushkov Institute of Cybernetic NASU (3 client +server CPU: Intel Xeon 2.67GHz, 32bit, memory: 2GB, network bandwidth $750 \mathrm{Mb} / \mathrm{s}$ ) with different $N_{\text {perone }}$ sent genomes are given in table 2. Because of Ethernet time delays for network $N_{\text {perone }}$ should be increased, whereas for cluster systems clients should be given by one genome on one step (thus processors which have calculated their task faster will be given another task to solve).

Table 2. Time of parallel calculations

\begin{tabular}{|c|c|c|c|c|}
\hline \multirow{2}{*}{ System } & \multicolumn{4}{|c|}{$\begin{array}{c}\text { Time of calculation (s) } \\
\text { with } N_{\text {peone }}:\end{array}$} \\
\cline { 2 - 5 } & $\mathbf{1}$ & $\mathbf{5}$ & $\mathbf{1 0}$ & $\mathbf{2 0}$ \\
\hline Cluster & 0.34 & 0.37 & 0.38 & 0.48 \\
\hline $\begin{array}{c}\text { PC (qnet) } \\
\text { network }\end{array}$ & 0.98 & 0.93 & 0.88 & 0.88 \\
\hline
\end{tabular}

\section{CONCLUSIONS}

The performance degradation of antenna arrays with elements failures may be partially compensated by a redistribution of the amplitude and phase over remaining working elements. Canonical genetic algorithm based approach provide stable, universal, but computationally hard approach to optimize antenna via power redistribution.

A CHC based method is proposed for the adaptation of antenna array with failed elements. The developed CHC algorithm provides stable and computationally easier approach to synthesize the amplitude and phase redistribution than canonical genetic algorithm.

Parallelization of CHC is easily implemented, but its optimal parameters depend on computational system type: for parallel computer it will be better to balance task between processor (i.e. to decrease number of sent to one CPU genomes to one), but for PC network because of transfer delays number of sent genomes doesn't influence a lot.

\section{REFERENCES}

[1] Peters T.J. A conjugate gradient-based algorithm to minimize the side lobe level of planar antenna arrays with element failures // IEEE Trans. on Antennas and Propagation, vol. 39, No. 10. 1991. p 1497-1504.

[2] Levitas M., Horton D.A., Cheston T.C. Practical Failure Compensation in Active Phased Array // IEEE Trans. on Antennas and Propagation, 1999. vol. 47 no. 3. p. 524-535.

[3] Yeo B., Lu Y. Array Failure Correction with Genetic Algorithm // IEEE Trans. on Antennas and Propagation, vol. 47. no. 5. 1999. p. 823828.

[4] Artyushenko B. Genetic Algorithm for Antenna Array with Failed and Deviated Elements Optimization // Proc. of IEEE Int. Workshop on Intelligent Data Acquasation and Advanced Computing Systems: Technology and Application (IDAACS'07) - Dortmund, Germany, 6-8 September 2007. pp. 228-231.

[5] Eshelman L. The CHC Adaptive Search Algorithm // Foundation of Genetic Algorithms, G. Rawlings, ed. Morgan-Kaufmann, 1991. p. 256-283.

[6] Gordon V.S., Whitley D. Serial and parallel genetic algorithms as function optimizers //Proceedings of the Fifth International Conference on Genetic Algorithms ed. Morgan Kaufman. 1993. pp. 177-183

[7] Gostyuhin A.V. Restorations of departure characteristics of antenna array with failed elements: PhD Thesis, Moscow, 2005, p. 125. In Russian. 


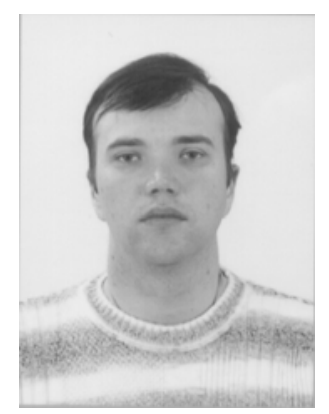

Artyushenko Bogdan graduated Computer System and Networks, Zaporizhzhya National Technical University.

PhD student in computer aided design.

Scientific interests: computer aided design, parallel computing, evolution strategy.

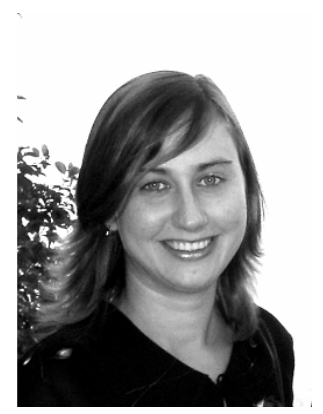

Shilo Galina graduated Software Engineering, Zaporizhzhya National Technical University. PhD, Assistant Professor.

Scientific interests: computer aided design, interval computations.

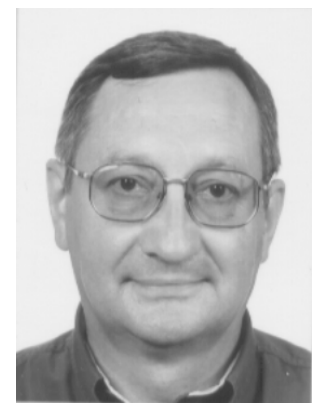

Krischuk Volodymyr graduated Radio Engineering, Zaporizhzhya National Technical University. PhD, Professor.

Scientific interests: computer aided design in electronics. 